\title{
Oscillations of rapidly rotating stars
}

\author{
D. Reese, F. Lignières, M. Rieutord
}

Laboratoire d'Astrophysique, Observatoire Midi-Pyrénées, Université Paul Sabatier Toulouse 3, 14 avenue É. Belin, 31400 Toulouse, France

\begin{abstract}
The effects of rapid rotation on stellar pulsation is examined using an accurate $2 \mathrm{D}$ numerical method. We compare the results of these non-perturbative calculations with those of perturbative methods and find that frequency differences exceed $0.08 \mu \mathrm{Hz}$ on half of the modes when the rotation rate is $15 \%$ of the keplerian (break-up) limit. The differences between the two results is mainly attributed to the approximate treatment of the centrifugal force in perturbative methods. We also explore different levels of approximation for the Coriolis force.
\end{abstract}

\section{Introduction}

The pulsation of rotating stars is of astrophysical interest because of the many unresolved questions on the structure of rotating stars, and the potential information that can be deduced through asteroseismology. With the launch of space missions such as Corot, we can expect to obtain accurate measurements of pulsation frequencies some of which will be of rapidly rotating stars. It is therefore important to understand and accurately quantify the effects of rotation on stellar pulsations.

There are two main effects that lead to a modification of stellar oscillations. The first is the centrifugal force which affects pulsation modes in a direct way through the effective gravity and in an indirect way through the deformation of the star. The second is the Coriolis force, which only has an effect through the momentum equation. In both cases, couplings appear between the different spherical harmonics, which adds to the mathematical difficulty of the problem.

\section{Formalism}

We calculate adiabatic acoustic oscillations of a uniformly rotating polytropic model of a star, in which the polytropic index $N$ is 3 . This can be decomposed into two steps: first we calculate the equilibrium model, second we solve the linearised oscillation equations. The numerical method we employ for both steps consists of a direct 2D numerical approach. It is entirely spectral as it uses spherical harmonics in the horizontal direction and Chebyshev polynomials in the radial direction. We use the surface-fitting coordinates of Bonazzola et al. (1998).

In order to project the oscillation equations onto the spherical harmonic base we start by expressing the different unknowns as a sum of spherical harmonics multiplied by radial functions that need to be determined. These expressions are introduced into the oscillation equations, which are then projected onto the vectorial spherical harmonics (e.g. Rieutord 
1987). In this way, we obtain a highly coupled system of ordinary differential equations in terms of the radial coordinate $\zeta$, the solution of which gives the unknown radial functions. This system is discretised onto a Gauss-Lobatto grid associated with the Chebyshev polynomials and the resulting algebraic eigenvalue problem is solved numerically.

We have done different tests to verify the accuracy of the calculated frequencies. In the non-rotating case we obtain an agreement of $\Delta \omega / \omega \sim 10^{-7}$ with the frequencies of Christensen-Dalsgaard and Mullan (1994). Also, for particular modes calculated without the Coriolis force, we obtain an agreement of $\Delta \omega / \omega \sim 10^{-6}$ with Lignières et al. (2005) for rotation rates up to $0.59 \Omega_{K}^{e q}$, where $\Omega_{K}^{e q}=\left(G M / R_{e q}^{3}\right)^{1 / 2}$ ( $R_{e q}$ being the equatorial radius). If we deduce perturbative coefficients from our frequencies, we obtain an agreement of 2 percent and usually better with the coefficients of Saio (1981). Finally, we also compute a variational test and obtain an accuracy of $10^{-7}$ or better.

\section{Results and physical aspects}

When examining the pulsation frequencies, it is interesting to compare them to those obtained through perturbative methods, in order to find out up to what point perturbative methods are valid. In what follows, we will consider modes with a harmonic degree $\ell$ ranging from 0 to 3 , an azimuthal order $m$ going from $-\ell$ to $\ell$, and the radial order $n$ ranging from 1 to 6 (strictly speaking an oscillation mode is composed of a whole set of harmonic degrees; here $\ell$ refers to the harmonic degree of the non-rotating mode). We follow their frequencies from the non-rotating case to a rotation rate of $0.38 \Omega_{K}^{e q}$.

Figure 1 shows a comparison between complete numerical frequencies and $2^{\text {nd }}$ order perturbative ones (we calculate the perturbative coefficients using a least squares fit near $\Omega=0$ ). As can be seen from the figure, perturbative calculations lead to errors exceeding $1 \%$ when the rotation rate is higher than $0.28 \Omega_{K}^{e q}$. If we consider a star with a mass of $1.9 M_{\odot}$ and a polar radius of $2.3 R_{\odot}$, a typical $\delta$ Scuti star, and we if impose an error bar of $0.6 \mu \mathrm{Hz}$ (the accuracy of Corot's secondary program), we find that the mode $(n=6, \ell=2, m=0)$ is erroneous at a rotation rate of $0.12 \Omega_{K}^{e q}$ (which corresponds to an equatorial velocity $\left.v_{e q}=47 \mathrm{~km} \cdot \mathrm{s}^{-1}\right)$ and half the modes are incorrect beyond $0.24 \Omega_{K}^{e q}\left(v_{e q}=95 \mathrm{~km} . \mathrm{s}^{-1}\right)$. If instead we impose an error bar of $0.08 \mu \mathrm{Hz}$ (the accuracy of Corot's primary program), the $(n=6, \ell=2, m=0)$ mode is erroneous at $0.075 \Omega_{K}^{e q}\left(v_{e q}=30 \mathrm{~km} \cdot \mathrm{s}^{-1}\right)$ and half the modes are incorrect at $0.15 \Omega_{K}^{e q}\left(v_{e q}=60 \mathrm{~km} . \mathrm{s}^{-1}\right)$.

It is then interesting to try to ascertain where these errors come from. Figures 2 and 3 give some clues as what may cause these differences. In Fig. 2, we show the relative error $\left(\omega_{\text {pert }}-\omega\right) / \omega$ from perturbative methods as a function the rotation rate. As the radial order $n$ increases, so does the relative error. In fact this trend is quite general, as it applies for all values of $\ell$ and $m$. In Fig. 3, we show another comparison between perturbative calculations and complete ones. This time, however, the Coriolis force has been suppressed in both sets of calculations. Once more, the relative error increases with the radial order, which shows that the centrifugal force alone reproduces the same behaviour as in Fig. 2. By contrast, we know that the effects of the Coriolis force decrease as the frequency increases. Therefore the error resulting from the perturbative treatment of the Coriolis force is not expected to increase with the radial order. This suggests that the centrifugal force plays the dominant role in the differences between perturbative and complete calculations. Figure 1 confirms this conclusion: the dashed lines represent calculations in which the Coriolis force is neglected but the centrifugal force is treated in a non-perturbative way. They give a better approximation than the perturbative calculations.

One of the reasons that may explain the centrifugal force's dominant role in the differences between the two approaches is the approximate treatment by perturbative methods of the star's deformation. Perturbative methods typically only use the $\ell=0$ and $\ell=2$ harmonics to 


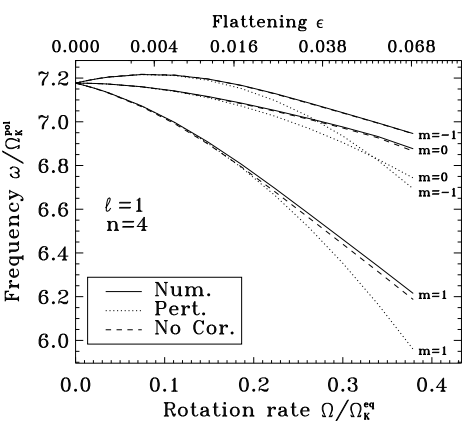

Figure 1: Non-dimensional frequencies as a function of the rotation rate. Solid lines: nonperturbative frequencies; dotted lines: $2^{\text {nd }}$ order perturbative frequencies; dashed lines: nonperturbative frequencies without the Coriolis force.

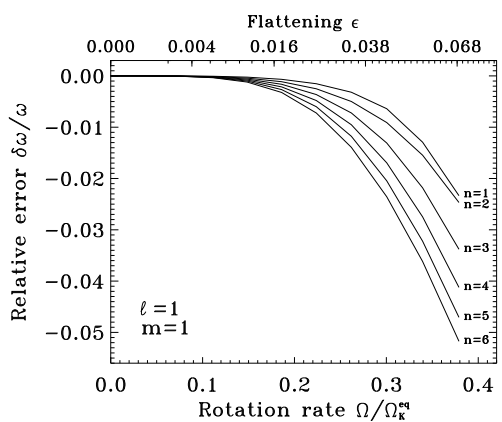

Figure 2: Behaviour of the relative error from $2^{n d}$ order perturbative methods as a function of the radial order $n$. The error increases with $n$. In all the figures, $\Omega_{K}^{e q}=\left(G M / R_{e q}^{3}\right)^{1 / 2}$, $\Omega_{K}^{p o l}=\left(G M / R_{p o l}^{3}\right)^{1 / 2}$ and $\epsilon=1-R_{p o l} / R_{e q}$, where $R_{e q}$ and $R_{p o l}$ are the equatorial and polar radii, resp.

describe this deformation, which is inaccurate, especially in the star's outer layers. Furthermore, differences between two descriptions of the deformation will give rise to differences in the frequencies which are roughly proportional to the frequencies. Hence a frequency twice as large as another will vary twice as much as the other due to modifications of the star's deformation. It is then helpful to bear in mind that as the radial order increases, pulsation frequencies also increase, and the associated modes become more concentrated in the star's outer layers. As a result, the centrifugal force rapidly becomes the dominant factor in the differences between the two approaches.

We can then take a more detailed look at the effects of the Coriolis force. In what follows, we will continue to treat the centrifugal force in a non-perturbative way and will look at three different ways of treating the Coriolis force. The first way consists in neglecting the Coriolis force. The second way corresponds to a first order perturbative approximation. The third way is a complete treatment. We denote these three levels of approximation by $\omega_{n \ell m}^{0}, \omega_{n \ell m}^{1}$ and $\omega_{n \ell m}^{2}$ respectively:

$$
\omega_{n \ell m}^{2}=\underbrace{\omega_{n \ell m}^{0}+D_{n \ell m} \Omega}_{\omega_{n \ell m}^{1}}+\mathcal{O}\left(\Omega^{2}\right), \quad \text { where } \quad D_{n \ell m}=\frac{i \int_{V} \rho_{o} \vec{e}_{z} \cdot\left(\vec{u}_{n \ell m} \times \vec{u}_{n \ell m}^{*}\right) d V}{\int_{V} \rho_{o}\left\|\vec{u}_{n \ell m}\right\|^{2} d V} .
$$

The expression for $D_{n \ell m}$ is very similar to the spherical expression (in both cases, the linear fluid dynamics operator is self-adjoint), but the integration domain is spheroidal, the equilibrium density is that of a rotating star and the eigenfunction $\vec{u}_{n \ell m}$ takes the centrifugal force into account, but not the Coriolis force. However, in the spherical limit, we obtain the familiar expression $D_{n \ell m}=m C_{n \ell}$ which can be found in Ledoux (1951).

Figure 4 shows the absolute errors of $\omega_{n \ell m}^{0}$ and $\omega_{n \ell m}^{1}$ for an $\ell=1$ triplet. In the $m=0$ case, $\omega_{n \ell m}^{0}=\omega_{n \ell m}^{1}$ since the corrections in that case start at second order in $\Omega$. For $m= \pm 1$, it is interesting to note that the first order correction $\omega_{n \ell m}^{1}$ brings the absolute error of both $m=1$ and $m=-1$ to roughly the same level (this implies increasing the error on $m=-1$ ). 


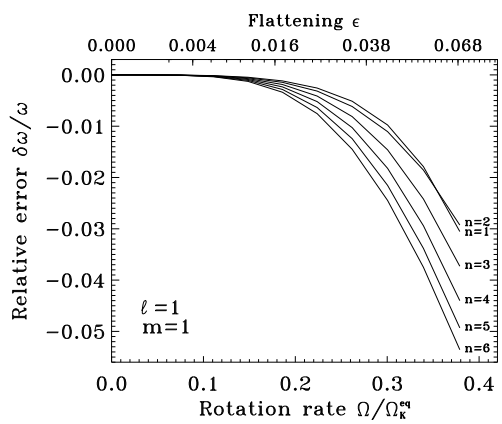

Figure 3: The relative error between complete numerical calculations and $2^{\text {nd }}$ order perturbative calculations, both of which exclude the Coriolis force. As $n$ increases, the error has the same behaviour as the calculations which include the Coriolis force (see Fig. 2).

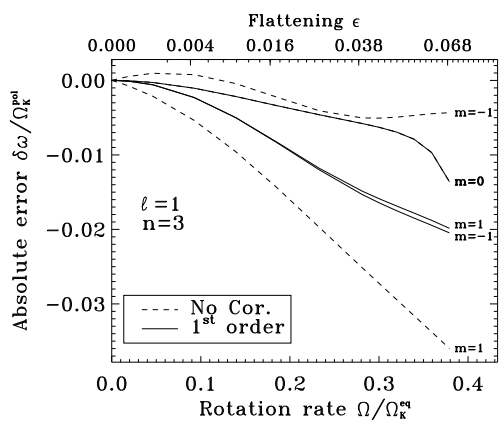

Figure 4: The absolute error caused by either neglecting the Coriolis force (No Cor.) or doing a $1^{\text {st }}$ order approximation of its effects (Pert.). The $1^{\text {st }}$ order approximation is not substantially better, but does make the errors symmetric in $m$, the same parity as $2^{\text {nd }}$ order corrections.

This shows that corrections symmetric in $m$, which come from even orders, are necessary. A second order correction might then adequately account for the Coriolis force.

\section{Conclusion}

In this paper, we have been able to examine some of the differences between a perturbative and a complete treatment of the effects of rotation on stellar pulsations, and explore the relative effects of the centrifugal and Coriolis forces. Our results show that the errors of perturbative methods can mostly be attributed to the approximate treatment of the centrifugal force.

\section{References}

Bonazzola, S., Gourgoulhon, E., Marck, J.A. 1998, Phys. Rev. D 58, 104020

Christensen-Dalsgaard, J., Mullan, D. J. 1994, MNRAS 270, 921

Ledoux, P. 1951, ApJ 114, 373

Lignières, F., Rieutord, M., Reese, D. 2005, in "Memorie della Societa' Astronomica Italiana", eds. A.R. Walker, G. Bono, Vol. 76/4, in press

Rieutord, M. 1987, Geophys. Astrophys. Fluid Dynamics 39, 163

Saio, H. 1981, ApJ 244, 299 